\title{
Summer Convective Afternoon Rainfall Simulation and Projection Using WRF Driven by Global Climate Model. Part I: Over Taiwan
}

\author{
Wan-Ru Huang ${ }^{1, *}$, Ya-Hui Chang ${ }^{1}$, Chao-Tzuen Cheng ${ }^{2}$, Huang-Hsiung Hsu ${ }^{3}$, Chia-Ying Tu ${ }^{3}$, \\ and Akio Kitoh ${ }^{4}$ \\ ${ }^{1}$ Department of Earth Sciences, National Taiwan Normal University, Taipei City, Taiwan, R.O.C. \\ ${ }^{2}$ National Sciences and Technology Center for Disaster Reduction, New Taipei City, Taiwan, R.O.C. \\ ${ }^{3}$ Research Center for Environmental Changes, Academia Sinica, Taipei City, Taiwan, R.O.C. \\ ${ }^{4}$ Life and Environmental Sciences, University of Tsukuba, Tsukuba, Japan
}

Received 22 May 2015, revised 20 March 2016, accepted 2 May 2016

\begin{abstract}
This paper is the first of a two-part study that investigates summer convective afternoon rainfall (CAR) activity over the East Asian continent through simulation and projection. We focus on the CAR activity over Taiwan in Part I. In Part II, the changes in CAR activity over South China and Luzon are examined. Using the WRF (Weather Research and Forecasting) model driven by two super-high resolution global climate models: HiRAM (High Resolution Atmospheric Model) and MRI (Meteorological Research Institute Atmospheric General Circulation Model), this study evaluates the performance of models (HiRAM, MRI, and WRF) used to simulate the summer CAR activity over Taiwan. The evaluations focus on the spatialtemporal variations in CAR activity during two time periods: the present-day (1979 - 2003, historical run) and the future (2075 - 2099, RCP8.5 scenario). For the present-day simulations, analyses indicate that both HiRAM and MRI cannot accurately simulate the temporal evolution of diurnal rainfall. This timing shift problem can be fixed after dynamical downscaling using WRF. The WRF dynamical downscaling approach also helps generate a more realistic CAR amount simulation over western Taiwan, where most residents live. In future projections all models predict that a significant decrease in CAR amount will occur over southwest Taiwan. This decrease in CAR amount is suggested due to the decrease in CAR frequency, not a change in the CAR rate. All models suggest that the cause of decrease in CAR frequency over southwest Taiwan is weaker local afternoon surface wind convergence and thermal instability to suppress CAR genesis.
\end{abstract}

Key words: Dynamical downscaling, Model simulations, Local rainfall, Taiwan

Citation: Huang, W. R., Y. H. Chang, C. T. Cheng, H. H. Hsu, C. Y. Tu, and A. Kitoh, 2016: Summer convective afternoon rainfall simulation and projection using WRF driven by global climate model. Part I: Over Taiwan. Terr. Atmos. Ocean. Sci., 27, 659-671, doi: 10.3319/TAO.2016.05.02.01

\section{INTRODUCTION}

Taiwan is a mountainous subtropical island located in the East Asian summer monsoon region (Fig. 1a). During summer (June, July, and August, or hereafter JJA), the rainfall in Taiwan is modulated by various types of weather systems (Chen and Chen 2003). Among these weather systems, convective afternoon rainfall (hereafter, CAR) is the most frequently observed pattern (Wang and Chen 2008). Because of its importance to local residents, the characteristics and causes of CAR have been examined in many studies (Chen et al. 1991; Johnson and Bresch 1991; Kerns et al. 2010;

\footnotetext{
* Corresponding author

E-mail:wrhuang@ntnu.edu.tw
}

Lin et al. 2011; Huang and Chan 2012; Wang et al. 2013). Studies have noted that CAR generally occurs on a day associated with a stronger sea breeze circulation during the daytime (e.g., Lin et al. 2012). Such a temporal sea breeze circulation evolution is found to be consistent with the local temperature change (Huang and Wang 2014). These observational studies focused only on the CAR change in the past or present day, rather than projections for future change.

Since global model simulations of future climate change are available, increasing attention has been given to how local climate will likely change in the future (e.g., Liang et al.2008). However, because the horizontal resolution of most global model simulations is generally too coarse to represent the details for local climate, many studies (e.g., 
Huang et al. 2013) have used the regional climate model to dynamically downscale the global climate simulation results to reproduce the local climate change, as reviewed by Hong and Kanamitsu (2014), Lee and Hong (2014), and Xue et al. (2014). Lin et al. (2015) recently examined the future change in local temperature in Taiwan projected by a nested regional-global climate model with Intergovernmental Panel on Climate Change (IPCC) A1B warming scenario. They noted significant temperature differences existed between the present (1979 - 2003) and future (2075 - 2099). Considering that the change in local temperature is one of the important factors for modulating CAR formation (Huang and Chen 2015), it can be inferred from Lin et al.'s (2015) finding that the CAR activity over Taiwan likely also presents significant differences between the present and future. The main objective of this study is to clarify this under-studied issue, with a focus on how CAR's characteristics and related maintenance mechanisms might be changed in the future.

A nested regional-global climate model approach has frequently been used to project future changes in regional climate (e.g., Liang et al. 2008; Lin et al. 2015), especially for the areas over complex terrain (e.g., Hong and Kanamitsu 2014; Lee and Hong 2014; Lee et al. 2014; Xue et al. 2014).
In this study, we use the Weather Research and Forecasting (WRF) model (Skamarock et al. 2008), a limited-area model, driven by two high-resolution global climate models: HiRAM (High Resolution Atmospheric Model; Zhao et al. 2009) and MRI (Meteorological Research Institute Atmospheric General Circulation Model; Mizuta et al. 2012), to project the future changes in CAR over Taiwan. The remainder of this manuscript is organized as follows. Details of the models, observational data and the statistical methods used for analyses are introduced in section 2 . Results are presented in section 3 , followed by a conclusion given in section 4 .

\section{MODELS, OBSERVATIONAL DATA, AND STATISTICAL METHODS}

\subsection{Models}

The HiRAM (Zhao et al. 2009) used in this study was developed by the Geophysical Fluid Dynamics Laboratory with a goal of providing improved representation of weather events in the global climate model. The HiRAM's horizontal resolution is approximately $25-\mathrm{km}$ over the East Asian summer monsoon region, coupled with 32 vertical layers. In contrast to HiRAM, the MRI (Mizuta et al. 2012), which

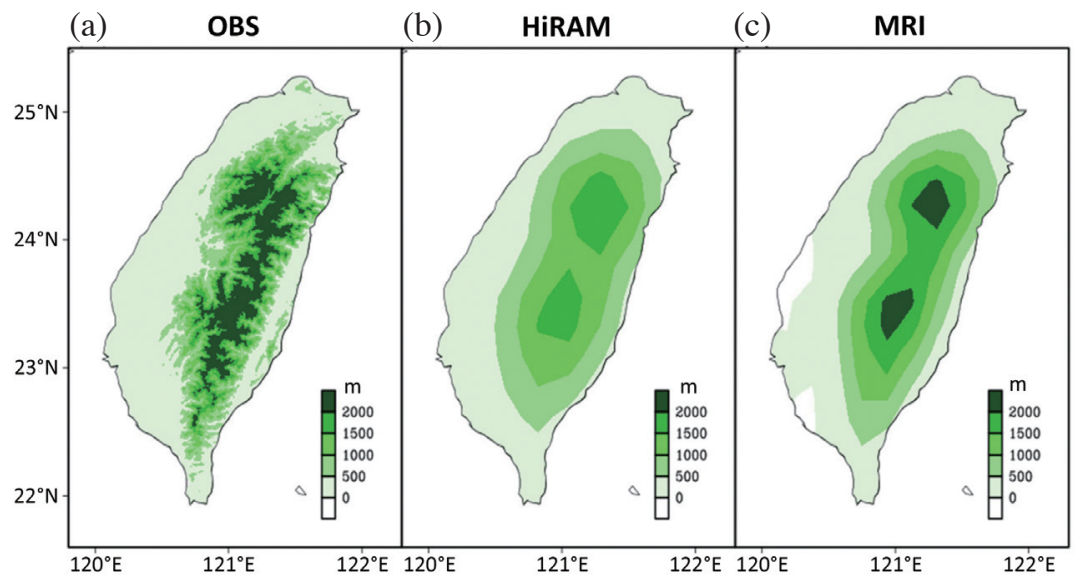

(d)

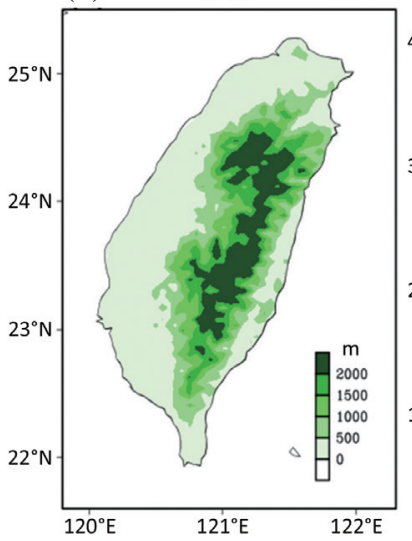

(e) Domain for downscaling using WRF

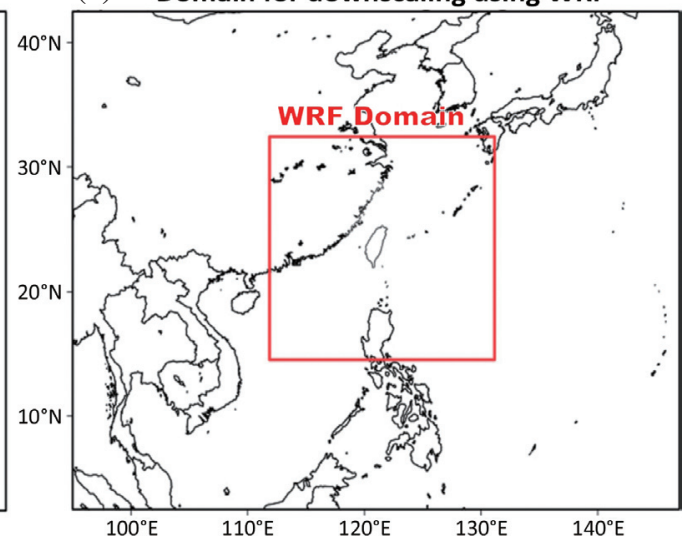

Fig. 1. Topography of Taiwan: (a) observation, (b) HiRAM, (c) MRI, and (d) WRF. (e) Geographical location of Taiwan and domain selected for WRF dynamic downscaling simulation (red boxed domain). 
was jointly developed by the Japan Meteorological Agency and the Meteorological Research Institute, has a horizontal resolution of approximately $20-\mathrm{km}$, with 64 vertical layers from the surface to $1 \mathrm{hPa}$. Both HiRAM and MRI use sea surface temperature and sea ice projected by the CMIP5 (Coupled Model Inter-comparison Project phase 5) multimodel ensemble dataset to drive the model. For additional HiRAM and MRI details please refer to Zhao et al. (2009) and Mizuta et al. (2012), respectively.

The analyses focus is on two time-sliced climate projections for the present (1979 - 2003, under the historical run) and the end of the $21^{\text {st }}$ century (2075 - 2099, under the RCP8.5 scenario). The dynamical downscaling experiments use version 3.5.1 of the WRF (Skamarock et al. 2008) driven by HiRAM (denoted as WRF-HiRAM) and MRI (denoted as WRF-MRI). Over the simulated domain (red boxed area in Fig. 1e), the WRF horizontal simulation resolution is $5-\mathrm{km}$ interval with 36 vertical layers. Because there is only one domain, no one-way or two-way nesting issue is considered in this study. In the WRF the planetary boundary layer simulations use the Noah land surface model (Tewari et al. 2004), the Yonsei-University boundary layer scheme (Hong et al. 2006), and the Monin-Obukhov surface layer scheme (Monin and Obukhov 1954). The microphysics parameterizations use the WRF Single-Moment five-class schemes (Hong et al. 2004). The CAM3 [version 3 of National Center for Atmospheric Research (NCAR) Community Atmosphere Model] radiation scheme (Collins et al. 2004) is used and the RCP8.5 Greenhouse Gas (GHG) concentration is considered in longwave radiation calculation. No cumulus parameterizations are applied. Spectrum nudging is only applied to atmospheric conditions, but not to the boundary layer.

\subsection{Observational Data}

The observational precipitation uses a $5-\mathrm{km}$ resolution uniform grid dataset, which is a remapping data from the Central Weather Bureau (CWB) rain-gauge-observations over Taiwan, provided by the Taiwan Climate Change Projection and Information Platform (TCCIP, http://tccip. ncdr.nat.gov.tw/v2/index.aspx). This 3-hourly gridded data is only available for the time period after 1992 because of the Taiwan rain gauge density during 1979 - 1991 was not sufficient for generating reasonable remapping data. Only the data during 1992 - 2003 is adopted to compare with the present-day simulations (1979 - 2003).

\subsection{Statistical Methods}

The analyses are presented for local Taiwan time (LT), which is the universal time $+8 \mathrm{~h}$ (i.e., $08 \mathrm{LT}$ corresponding to 00 UTC). Based on the diurnal rainfall observational characteristics (Fig. 2a, to be discussed later), a CAR day is identified as when (1) the accumulated rainfall during 1200 - $2200 \mathrm{LT}$ is greater than $80 \%$ of the daily rainfall, (2) the accumulated rainfall during $0100-1100 \mathrm{LT}$ is smaller than $10 \%$ of the daily rainfall, and (3) the days affected by other weather systems (e.g., typhoons and frontal systems) are excluded. These criteria are applied for both the observation and the models. Statistical significances for the analyzed variables are determined using a two-tailed Student's t-test based on the effective degree of freedom (Von Storch and Zwiers 1999).

\section{RESULTS}

\subsection{Present-Day Simulations}

Figures 1a - d show the Taiwan topography extracted from the observation and the models. Among all models, WRF (Fig. 1d) presents the best illustration of Taiwan topography, showing plains and gentle slopes in the west, a central mountain range and steep slopes in the east (Fig. 1a).
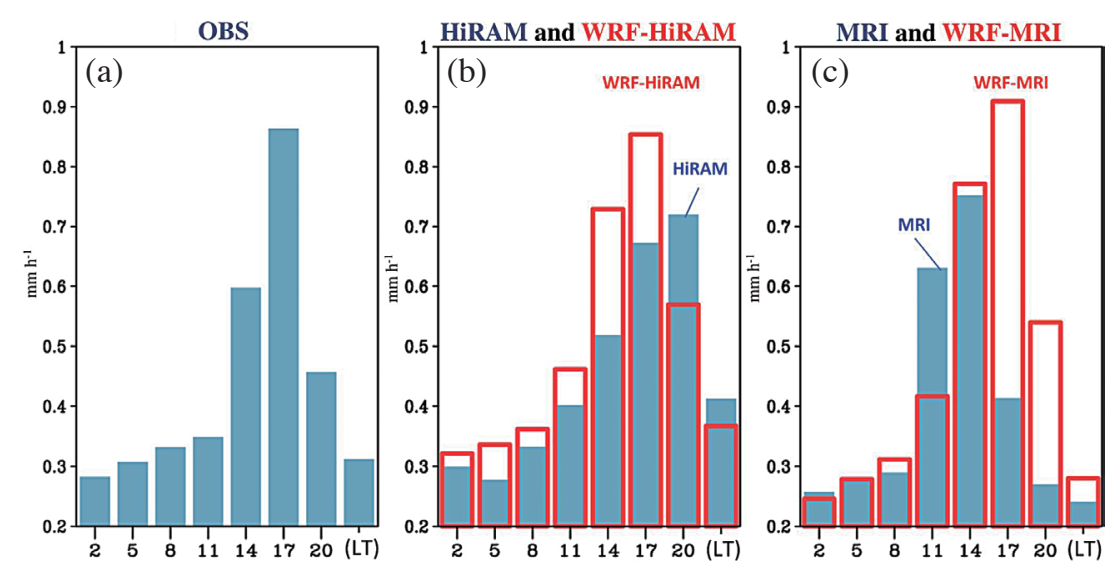

Fig. 2. Temporal evolution of climatological 3 hourly rain rate: (a) observation, (b) HiRAM (blue bar) and WRF-HiRAM (red bar), (c) MRI (blue bar) and WRF-MRI (red bar) area-averaged over Taiwan during summer. The estimation is based on the data extracted from surface observation during 1992 - 2003 and from model simulations during 1979 - 2003. 
For HiRAM (Fig. 1b) and MRI (Fig. 1c), the terrain structure is too smooth and the high mountain altitude is overall lower than the observation. Based on these features and considering that the diurnal rainfall formation characteristics over Taiwan are very dependent upon the geography (Huang and Wang 2014), it is likely that using the dynamical downscaling approach (i.e., WRF-HiRAM and WRF-MRI, with better representation of topography) is more suitable than using HiRAM or MRI alone in simulating the diurnal rainfall over Taiwan.

To verify this hypothesis, we examine the temporal evolution of JJA mean 3-hourly rain rate, extracted from the rain-gauge observation (Fig. 2a) and model simulations (Figs. $2 \mathrm{~b}-\mathrm{c}$ ), and averaged by the area over Taiwan. Note from the observations (Fig. 2a) that the summer diurnal rainfall in Taiwan generally reaches its maximum value in the afternoon at 17 LT. By comparing this feature with that extracted from the HiRAM's simulation (blue bar in Fig. 2b), the results show that HiRAM tends to generate the maximum diurnal rainfall at $20 \mathrm{LT}$, about 3 hours later than the observation. By contrast, the MRI's simulated diurnal rainfall maximum (blue bar in Fig. 2c) generally appears at $14 \mathrm{LT}$, approximately 3 hours earlier than the observation. It is worth noting that even though the horizontal resolutions of HiRAM $(\sim 23 \mathrm{~km})$ and MRI $(\sim 20 \mathrm{~km})$ are high enough to resolve the land-sea breeze feature (i.e., $<100 \mathrm{~km}$ ), which is known as an important factor for modulating the diurnal rainfall formation over the East Asian coastal regions (Huang et al. 2010), both HiRAM and MRI still cannot simulate well the diurnal rainfall evolution over Taiwan. As inferred from Fig. 1, part of the disability of HiRAM and MRI in simulating the diurnal rainfall evolution over Taiwan might be due to the non-realistic depiction of topography in the models, which likely leads to a less realistic physics representation in the global climate models at the regional scale (e.g., Lin et al. 2015). This finding is consistent with other studies suggesting that the global climate models generally have a timing shift problem in simulating diurnal rainfall (Chow and Chan 2009).

On the other hand, by comparing the WRF's simulated diurnal rainfall (red bars in Figs. $2 \mathrm{~b}$ - c) with the observation (Fig. 2a), the results show that both WRF-HiRAM and WRFMRI accurately depict the diurnal rainfall evolution, featured with a maximum value at $17 \mathrm{LT}$ and a minimum value at 02 LT. These features (Fig. 2) imply that the WRF dynamical downscaling approach can help reduce the biases in the diurnal rainfall variation simulation at regional scales from the driving global climate models. Considering that a proper simulation of diurnal rainfall formation is important for more realistic CAR event detection, it is expected that WRF-HiRAM and WRF-MRI also perform better than HiRAM and MRI on the simulation of CAR activity over Taiwan.

To clarify this inference, we examined the spatial distributions of mean accumulated CAR amount (i.e., average
CAR rainfall accumulated during a summer) estimated from the observation and model simulations (Fig. 3). Note that in order to evaluate which model (HiRAM, MRI or WRF) performs best in simulating CAR activity, the same criteria for CAR event identification were applied for all models (see section 2.3), despite the inter-model differences revealed in Fig. 2. Overall, the observation in Fig. 3 shows that the distribution of CAR amount is characterized by a clear eastwest contrast, with larger (smaller) values in the west (east) side of the mountain range. The formation of this asymmetric distribution might be attributed to the fact that the southwesterly monsoon wind generally brings more moist and unstable air for CAR formation over western Taiwan (i.e., windward side) (e.g., Ruppert et al. 2013; Huang and Wang 2014). Different from the observation, the HiRAM's simulated CAR amount distribution generally follows the terrain structure (i.e., larger values centered at the high mountains and smaller values at the low-plains). In contrast, the MRI's simulation in Fig. 3 reveals an asymmetric pattern (i.e., larger CAR amount over western Taiwan than in other sub-regions) similar to the observation. However, because MRI tends to underestimate the diurnal rainfall during 1200 - 2200 LT (see Fig. 2c), the magnitude of MRI's estimated CAR amount is much smaller than the observed value (see Fig. 3). As for WRF-MRI and WRF-HiRAM, both of their simulations on the distribution of CAR amount seem to be more realistic over the west side of the mountain ranges than in the other sub-regions.

To further clarify the models' ability to simulate CAR activity we also examined the spatial distribution of CAR's contribution to the total summer rainfall amount estimated from the observation and model simulations (Fig. 4). As revealed in Fig. 4, the observation shows that the CAR amount plays a more important role in contributing to the total summer rainfall over western Taiwan than over the other sub-regions. On average, about $40-50 \%$ of the total summer rainfall over western Taiwan is contributed by CAR events. This feature is well simulated by the WRFHiRAM and WRF-MRI, but underestimated by the HiRAM and MRI. In contrast, for most of eastern Taiwan, where the observation shows that only $20-30 \%$ of the total summer rainfall is contributed by CAR events, both WRF-HiRAM and WRF-MRI tend to overestimate CAR's contribution to the total summer rainfall.

Statistical evidence supporting the findings in Figs. 3 - 4 is given in Fig. 5. In Fig. 5, we separate Taiwan into four different sub-regions based on the topography characteristics and the overall features revealed in Figs. 3 - 4. From Fig. 5, it is clear that using the WRF dynamical downscaling approach can help generate a more realistic simulation on the CAR activity over the west side of the mountain range (including sub-regions 1 and 2), but perhaps not over the other sub-regions (including sub-regions 3 and 4). According to this finding, the reminder of the examinations will focus on 


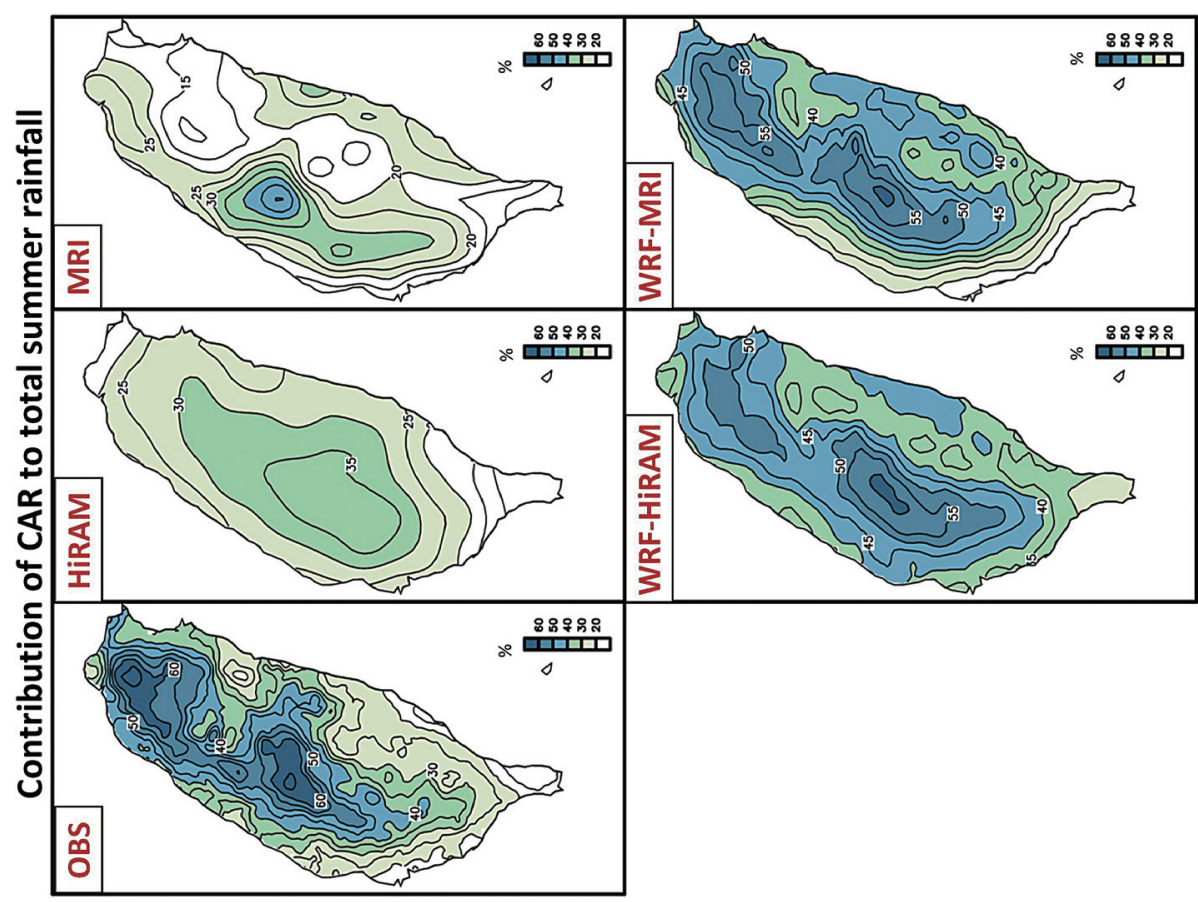

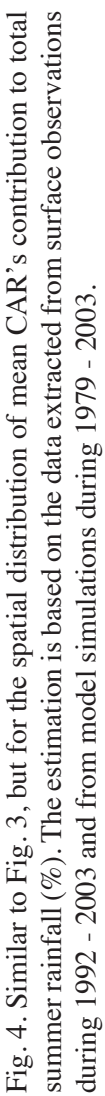

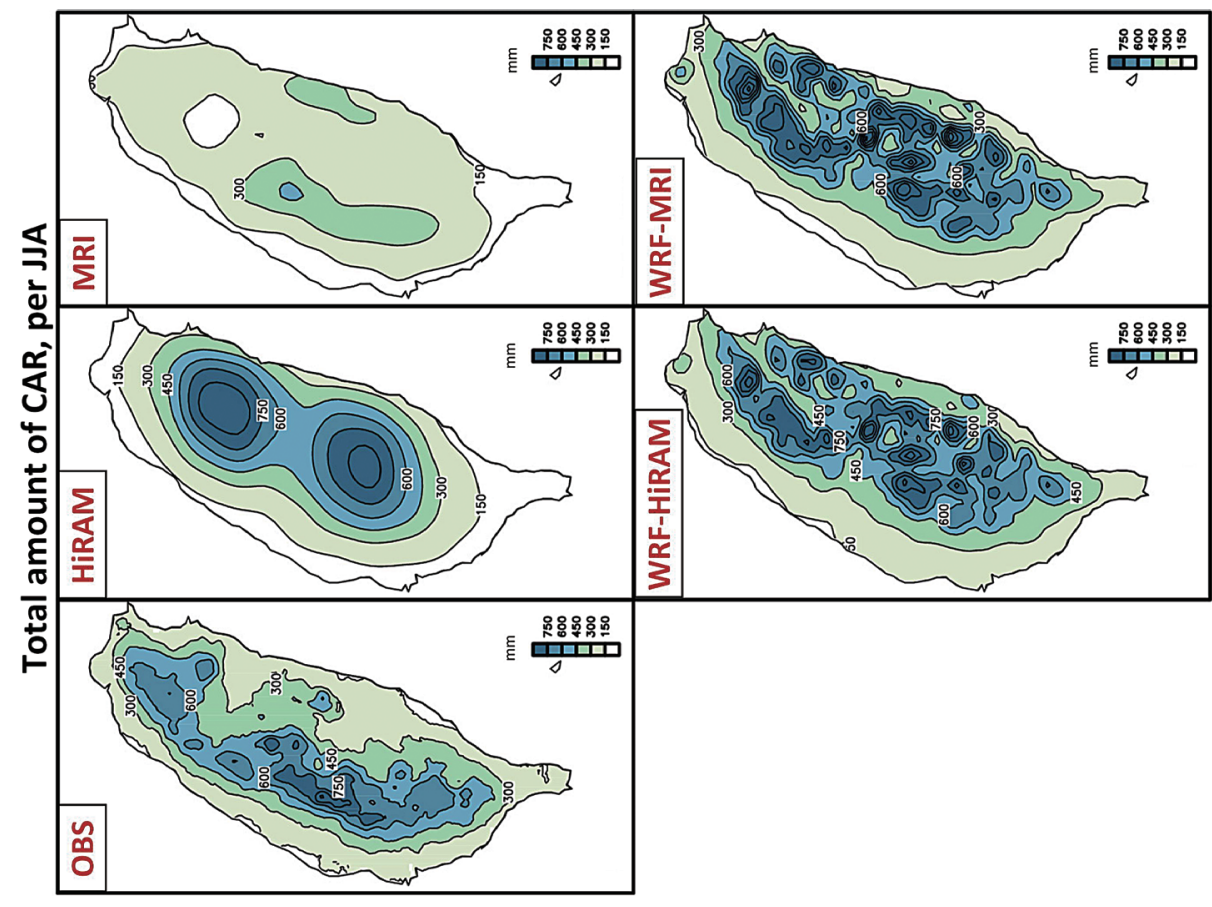

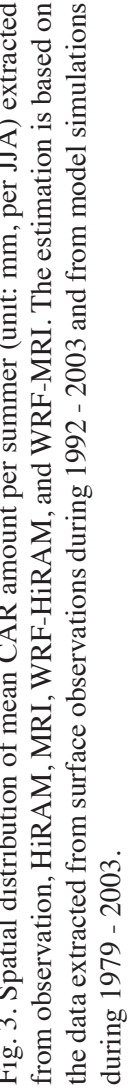


(a)

\section{Total amount of CAR, per JJA}

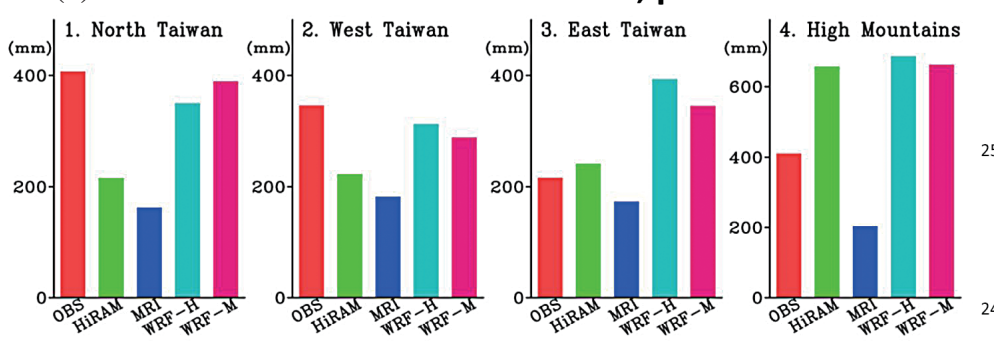

(b) Contribution of CAR to total summer rainfall
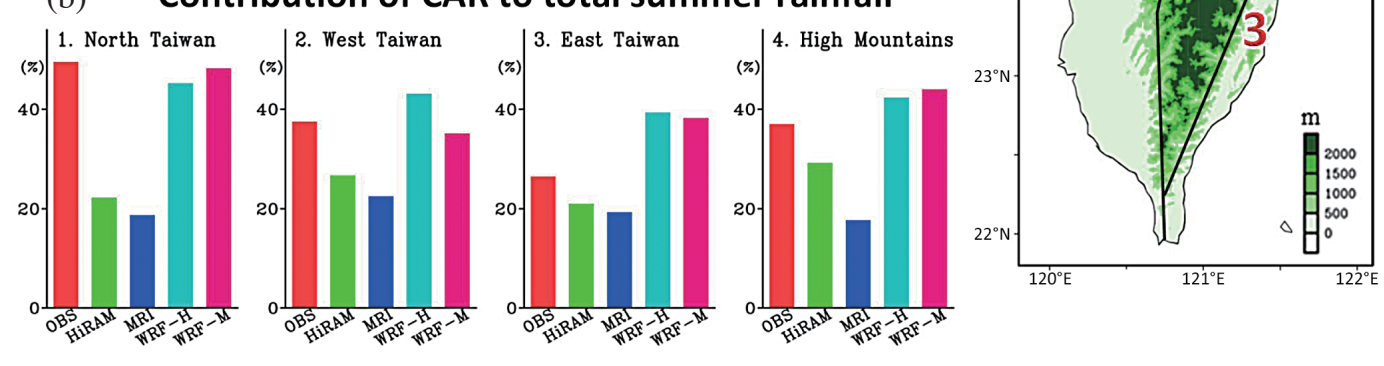

Fig. 5. Area-averaged values of (a) CAR amount (unit: mm, per summer) and (b) CAR's contribution to total summer rainfall (unit: \%) over four Taiwan sub-regions identified in the right panel. The data for (a) - (b) were extracted from Figs. 3 and 4, respectively. WRF-H and WRF-M represents WRF-HiRAM and WRF-MRI, respectively.

issues related to future changes in CAR activity over the west side of the mountain range where most residents live.

\subsection{Future Projections}

We next examined the difference in CAR amount between two selected periods: 2075 - 2099 and 1979 - 2003 (i.e., 2075 - 2099 minus 1979 - 2003; see Fig. 6) to project the future changes in CAR. As seen from Fig. 6 the HiRAM's projection indicates that the CAR amount will largely increase over the high mountains, but decrease over the western and southern plains. The MRI's projection shows that the CAR amount will decrease significantly over west Taiwan (i.e., sub-region 2 in Fig. 5), but increase over north and east Taiwan. The WRF-HiRAM and WRF-MRI's projections suggest that the decrease in CAR amount will appear mainly over the areas covering the western slope of the mountain range and southwest Taiwan. Detailed information on the projected changes in CAR amount averaged over north Taiwan, west Taiwan, east Taiwan, and the high mountains (as defined in Fig. 5) are documented in Table 1 to help better illustrate the regional differences in CAR amount projection.

As revealed in Table 1, the models' projection of the changes in CAR amount is more consistent over west Taiwan than over the other sub-regions. Overall, all models suggest that the CAR amount will decrease significantly over southwest Taiwan (also see Fig. 6) in the future. Consistent with the features revealed in Fig. 6, all models also predict that the CAR's contribution to the total summer rainfall will decrease significantly over southwest Taiwan (see Fig. 7 and
Table 1). For most of eastern Taiwan, the results (see Fig. 7 and Table 1) show that the HiRAM and MRI's predictions for an increase in CAR's contribution to the total summer rainfall is opposite to that predicted by WRF-HiRAM and WRF-MRI (i.e., decrease).

What causes the projected decrease in CAR amount over southwest Taiwan? It is known that the variation in CAR amount is associated with the change in CAR frequency (i.e., the number of CAR days per JJA) and CAR rate (i.e., the average rain rate during a CAR day); CAR amount = CAR frequency $\times$ CAR rate. In order to clarify which factor (rain frequency or rain rate) plays a more important role in determining the projected change in CAR amount, we next examined the future change in CAR frequency and CAR rate (Figs. 8 - 9). For HiRAM, the projections show that the CAR frequency will decrease over the low-plains, but increase over the high mountains (Fig. 8). In contrast, the CAR rate will increase over most of Taiwan, except southeast Taiwan (Fig. 9). These features together with Fig. 6 imply that the HiRAM projected decrease in CAR amount over western Taiwan is induced mainly by the decrease in CAR frequency, not a change in the CAR rate. For MRI, both of its projections on the CAR frequency and CAR rate show a decrease will occur over most of west Taiwan. However, because more significant change is revealed in CAR frequency (i.e., more areas passing the significant test) than in CAR rate, it is also reasonable to infer from Figs. 8 - 9 that the MRI projected decrease in CAR amount over southwest Taiwan is contributed primarily by the decrease in CAR frequency and less by the decrease in CAR rate. Both the WRF-HiRAM and WRF-MRI projections show that CAR over most of Taiwan 
will become fewer (see Fig. 8) but stronger (see Fig. 9). These features together with Fig. 6 suggest that WRF-HiRAM and WRF-MRI's projected decrease in CAR amount is due to the decrease in CAR frequency, not the change in CAR rate.

From the above discussions, it is clear that all models suggest that the future decrease in CAR amount over southwest Taiwan is caused mainly by the decrease in CAR frequency. The next question is what causes the projected decrease in CAR frequency over southwest Taiwan? Previous studies (e.g., Lin et al. 2012; Huang and Wang 2014) examining the observational data suggested that the increase in daytime low-level wind convergence could provide more "dynamic" lift to facilitate CAR genesis. Using WRF-HiRAM and WRF-MRI as examples, the present-day simulations (Fig. 10a) show that the daytime near-surface wind over Taiwan is mainly modulated by: (1) sea-breeze convergence, (2) upslope flow, and (3) orographic lifting of prevailing southwesterly flow (e.g., Johnson and Bresch 1991). By examining the difference between the future and present-day near-surface wind change at 14 LT (Fig. 10b), further results indicate that over west Taiwan (i.e., the plain area) the change in near-coastal wind (i.e., sea-breeze) is much larger than the change in near-mountain wind (i.e., upslope flow or orographic lifting of prevailing flow). Similar features can be also revealed from the HiRAM and MRI simulations (not shown); suggesting that the change in nearsurface wind convergence over west Taiwan is mainly contributed by the change in sea-breeze convergence.

To clarify, if the change in sea-breeze convergence (i.e., dynamic lifting) can be applied to explain the change in CAR frequency over western Taiwan in the future, we examine the projected change in the near-surface wind convergence at 14 LT [i.e., the time step with maximum diurnal surface wind convergence over Taiwan (Huang and Wang 2014)] (as in Fig. 11). The method applied for generating Fig. 11 is described as follows. It should be noted that the calculation of near-surface wind convergence by $10 \mathrm{~m}$ wind in the highresolution model might present a notable problem over steep and complex terrain (i.e., while the surface winds are not at a similar height level). To deal with this problem we first remove the gridded data over the high mountain areas. We then apply the Cressman objective analysis (Cressman 1959) to interpolate the rest of the gridded data into a user-defined latitude-longitude grid for the entire Taiwan area. By doing this the new gridded data can be seen at a similar height level and used to calculate the near-surface wind convergence over Taiwan. Considering the reality of sea-breeze convergence, which does not exist over the interior Central Mountain Range, the mountainous area in Fig. 11 is blocked.

As revealed in Fig. 11 all models project that the daytime surface wind convergence will decrease over most of western Taiwan. Such a change in daytime surface wind convergence implies a weaker dynamic lifting in the future. This change in atmospheric conditions would likely sup- press the chance for CAR formation over western Taiwan, as revealed in Fig. 8. Note also from Fig. 11 that all models predict that the daytime surface wind convergence will increase over most of eastern Taiwan. Such an increase in surface wind convergence over eastern Taiwan is induced mainly by the intensification of orographic lifting of southerly wind, as inferred from Fig. 10b. This finding on the change of "dynamic" lifting, however, cannot explain why the projected change in CAR frequency over most of eastern Taiwan is not consistent among the models (see Fig. 8). One possible reason is that the change in daytime surface wind convergence is not the only factor important for the modulation of CAR frequency. In fact, it can be inferred from other observational studies (e.g., Huang and Chen 2015; Huang et al. 2015) that the change in thermal instability also has a significant impact on the modulation of CAR frequency. Based on this suggestion, we next examined the projected change in thermal instability (as in Fig. 12) to clarify its role in modulating the future changes in CAR frequency.

Figure 12a shows the projected change (i.e., 2075 2099 minus 1979 - 2003) in thermal instability [i.e., -dT/dz (estimated from the temperature change with height)] at $14 \mathrm{LT}$. Considering the altitude of the highest mountain in Taiwan is close to $4000 \mathrm{~m}(\sim 600 \mathrm{hPa}$ level $),-\mathrm{dT} / \mathrm{dz}$ in Fig. 12a is estimated from the temperature change between the surface and $600 \mathrm{hPa}$. As inferred from Fig. 12a, all models predict that the thermal instability will decrease in the future [i.e., - dT/dz (future - present) < 0] for most of Taiwan. Such a decrease in thermal instability suggests that fewer thermally induced CAR events will likely occur in the future for the most of Taiwan. This decrease in thermal instability also contributes to the decrease in CAR frequency over western Taiwan (e.g., Huang et al. 2015). As for eastern Taiwan, where the dynamically induced CAR frequency increases (as inferred from Figs. 10 - 11) but thermally induced CAR frequency decreases (as inferred from Fig. 12), the thermal factor seems to be more important than the dynamic factor in determining the change in CAR frequency in the WRF model (see Fig. 8). While for HiRAM and WRF, it is likely that the dynamic factor plays a more important role than the thermal factor in modulating the change in CAR frequency, these suggestions require more supporting evidence in the future study.

Note that the projected change in thermal instability revealed in Fig. 12a is found to be induced by a larger increase in the higher-level temperature and a smaller increase in the lower-level temperature (Fig. 12b). This finding in future temperature change over Taiwan is consistent with the findings of Lin et al. (2015), showing that the model projects future warming in the high mountains will be larger than in the low-plains. Summarized from Figs. 10 - 12, it is suggested that both the change in daytime surface wind convergence and the change in thermal instability should be considered together when explaining the future change in 
CAR frequency over Taiwan. Notably, the causes of future change in CAR rate over Taiwan were not examined in this study because the projected change in CAR rate is less significant and less consistent among the models (see Fig. 9).

\section{CONCLUSION}

This study examined the present-day simulation and future projection of CAR change over Taiwan using WRF driven by two super-high resolution models: HiRAM and MRI. For the present-day simulations, the analyses indicate that both HiRAM and MRI have problems in simulating the right diurnal rainfall evolution, but this timing shift problem can be corrected after dynamical downscaling using WRF driven by HiRAM (i.e., WRF-HiRAM) and MRI (i.e., WRFMRI) (see Fig. 2). The analyses also indicate that, relative to HiRAM and MRI, both WRF-HiRAM and WRF-MRI have a more realistic simulation for the CAR amount distribution

Projected change in CAR amount, per summer

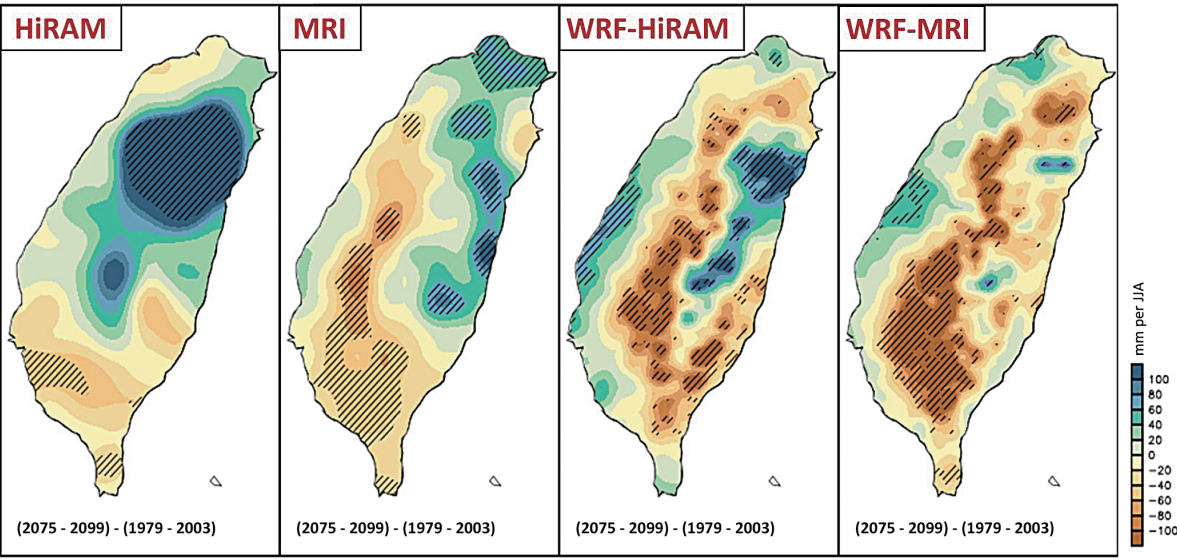

Fig. 6. Difference in JJA mean accumulated CAR amount between future (2075 - 2099) and present-day (1979 - 2003) simulations. The data were extracted from HiRAM, MRI, WRF-HiRAM, and WRF-MRI. The changes significant at the $90 \%$ confidence interval are marked.

Table 1. Difference between future (2075 - 2099) and present-day (1979 2003) simulation for CAR amount (Fig. 6, unit: mm, per JJA), CAR's contribution to total summer rainfall (Fig. 7, unit: \%), CAR frequency (Fig. 8, unit: days per JJA), and CAR rate (Fig. 9, unit: mm, per CAR days) area-averaged over four Taiwan sub-regions (as identified in Fig. 5).

\begin{tabular}{l|l|rrrr}
\hline \multicolumn{1}{l|}{} & & HiRAM & \multicolumn{1}{c}{ MRI } & WRF-HiRAM & WRF-MRI \\
\hline \multirow{4}{*}{ North Taiwan } & Fig. 6 & 10.67 & 36.68 & -12.24 & -4.06 \\
& Fig. 7 & 2.11 & 1.53 & -5.42 & -2.62 \\
& Fig. 8 & -4.93 & 0.84 & -5.55 & -5.59 \\
& Fig. 9 & 2.57 & 1.48 & 1.13 & 1.63 \\
\hline \multirow{5}{*}{ West Taiwan } & Fig. 6 & -7.45 & -18.40 & -16.56 & -12.76 \\
& Fig. 7 & -2.36 & -2.74 & -12.45 & -4.88 \\
& Fig. 8 & -7.91 & -1.61 & -4.71 & -4.86 \\
& Fig. 9 & 1.75 & -0.06 & 1.49 & 1.54 \\
\hline & Fig. 6 & 9.99 & 7.20 & -32.22 & -20.10 \\
& Fig. 7 & 2.40 & 1.20 & -2.71 & -3.05 \\
& Fig. 8 & 0.49 & -1.31 & -5.98 & -6.20 \\
& Fig. 9 & 0.51 & 0.52 & 1.14 & 1.15 \\
\hline \multirow{5}{*}{ High Mountains Taiwan } & Fig. 6 & 174.89 & 6.23 & -11.70 & -55.32 \\
& Fig. 7 & 4.31 & 1.43 & -5.91 & -6.07 \\
& Fig. 8 & 2.54 & 0.28 & -7.47 & -9.39 \\
& Fig.9 & 2.97 & -0.20 & 2.37 & 1.55 \\
\hline
\end{tabular}


Projected change in CAR's comtribution to total summer rainfall

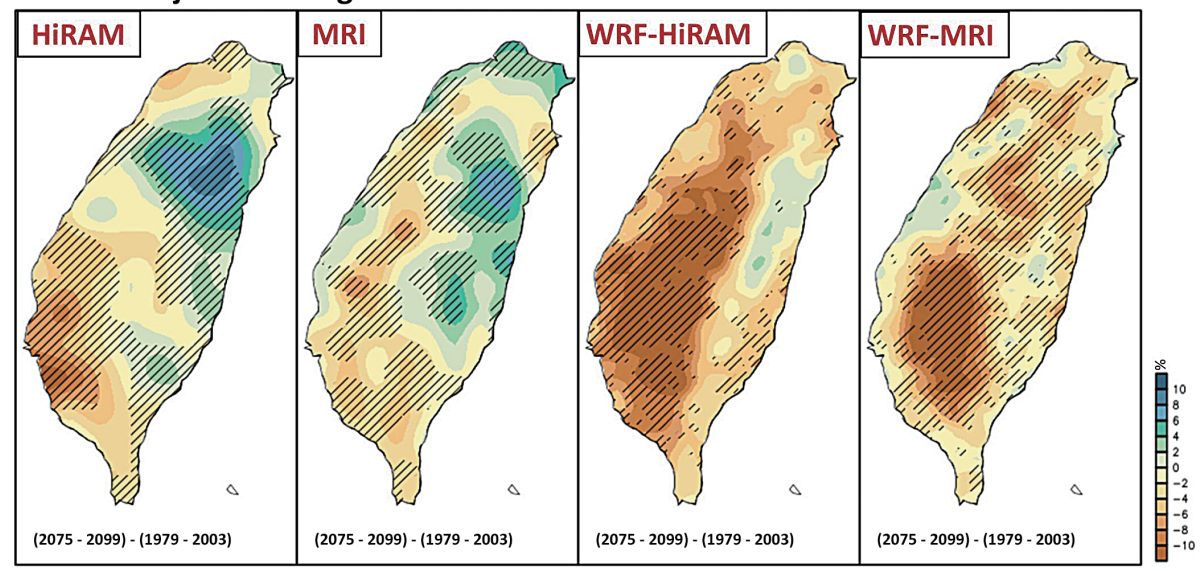

Fig. 7. Difference in mean CAR's contribution to total summer rainfall between future (2075 - 2099) and present-day (1979 - 2003) simulations. The data were extracted from HiRAM, MRI, WRF-HiRAM, and WRF-MRI. The changes significant at the $90 \%$ confidence interval are marked.

Projected change in CAR days, per summer

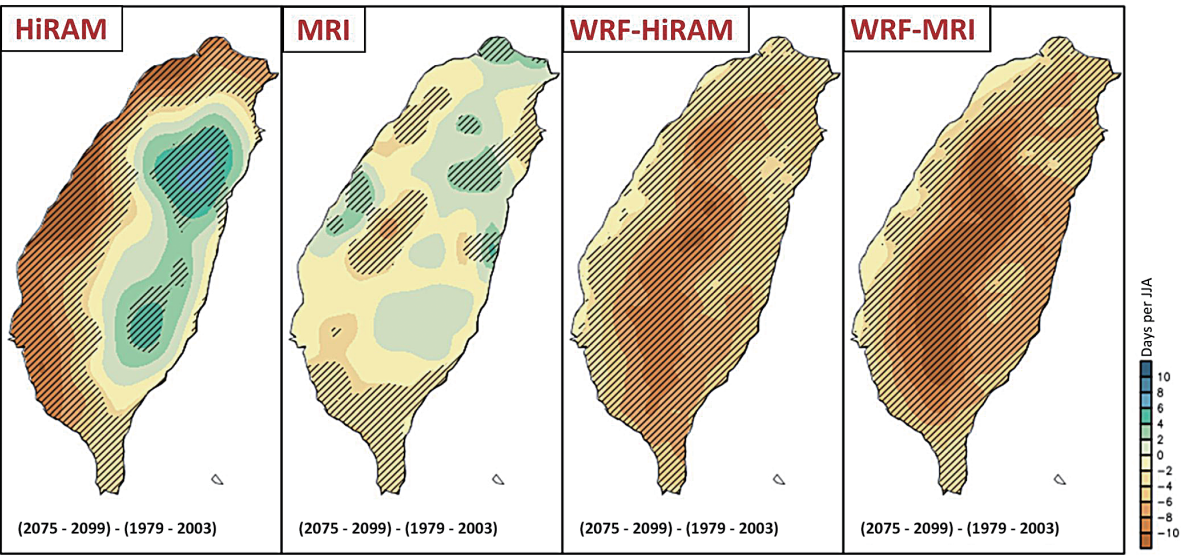

Fig. 8. Difference in CAR frequency (unit: average days per JJA) between future (2075 - 2099) and present-day (1979 - 2003) simulations. The data were extracted from HiRAM, MRI, WRF-HiRAM, and WRF-MRI. The changes significant at the $90 \%$ confidence interval are marked.

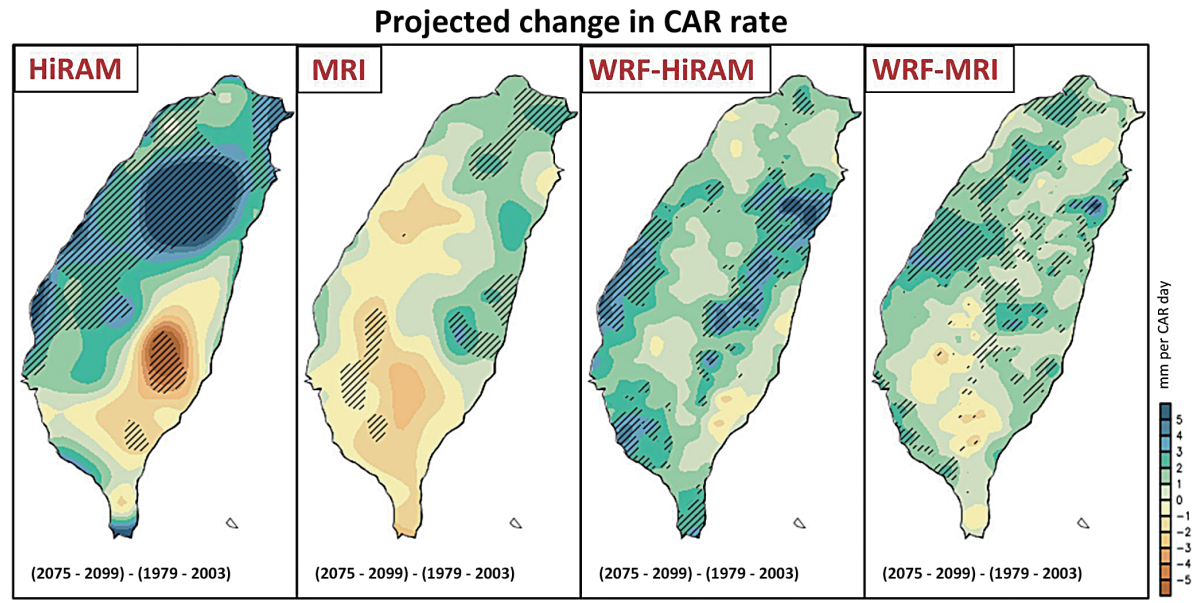

Fig. 9. Difference in CAR rate (unit: mm, per CAR days) between future (2075 - 2099) and present-day (1979 - 2003) simulations. The data were extracted from HiRAM, MRI, WRF-HiRAM, and WRF-MRI. The changes significant at the $90 \%$ confidence interval are marked. 
(a) Present-day simulation of Vs at 14 LT

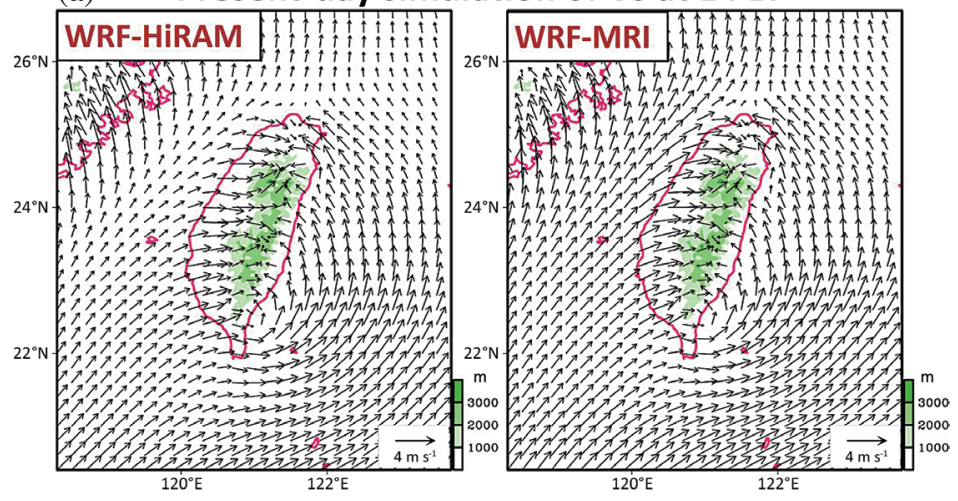

(b) Projected change in Vs at 14 LT

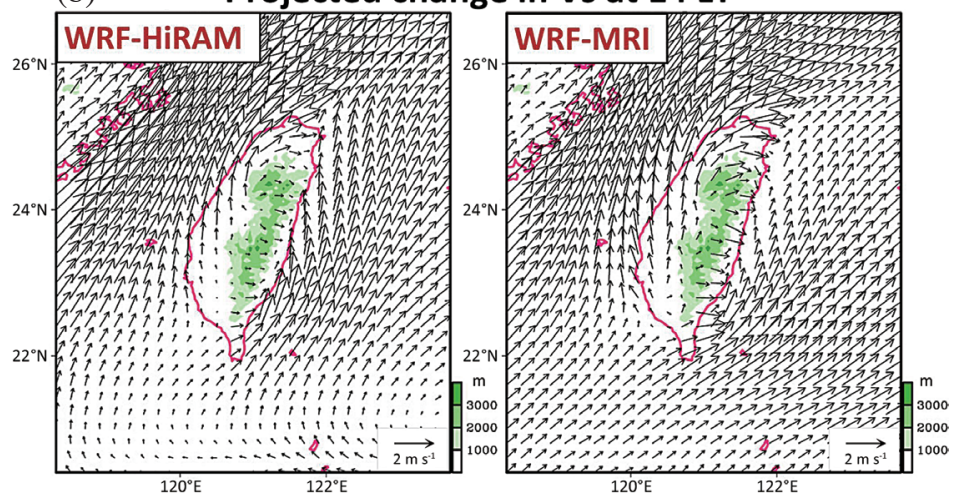

Fig. 10. (a) Present-day (1979 - 2003) simulations for near-surface wind at 14 LT. (b) Difference between future (2075 - 2099$)$ and present-day (1979 - 2003) simulations for near-surface wind at 14 LT. The data were extracted from WRF-HiRAM and WRF-MRI. The mountain areas are shaded.

Projected change in $(-\nabla \cdot V s)$ at $14 \mathrm{LT}$

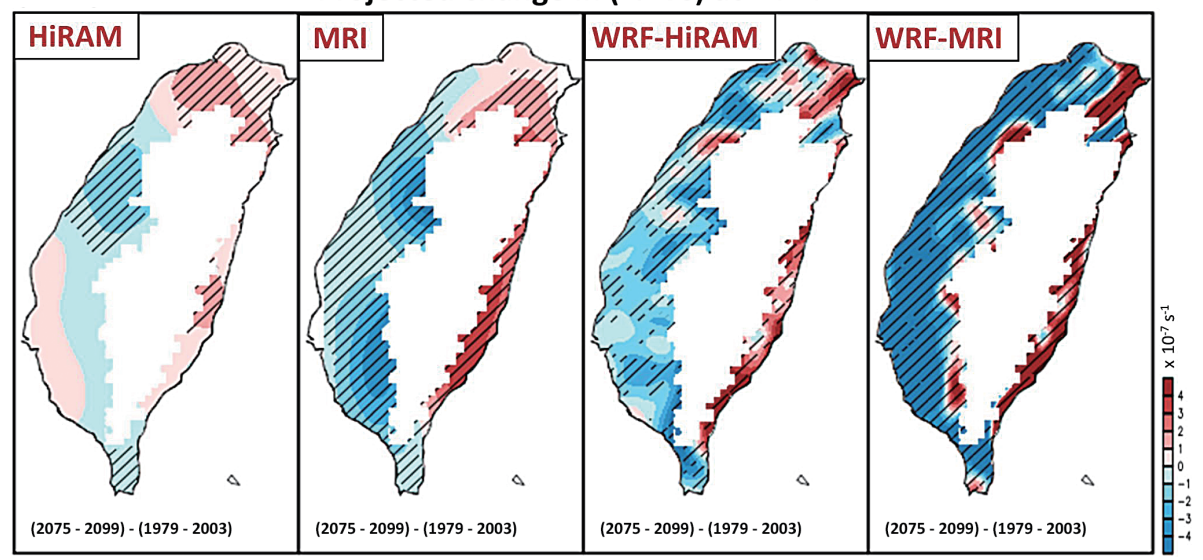

Fig. 11. Difference between future (2075 - 2099) and present-day (1979 - 2003) simulations for near-surface wind convergence [i.e., (- $\nabla \cdot V s)]$ at 14 LT. The data were extracted from HiRAM, MRI, WRF-HiRAM, and WRF-MRI. The changes significant at the $90 \%$ confidence interval are marked, and the mountain areas are blocked. 
(a) Projected change in thermal instability, i.e., $-d T / d z$

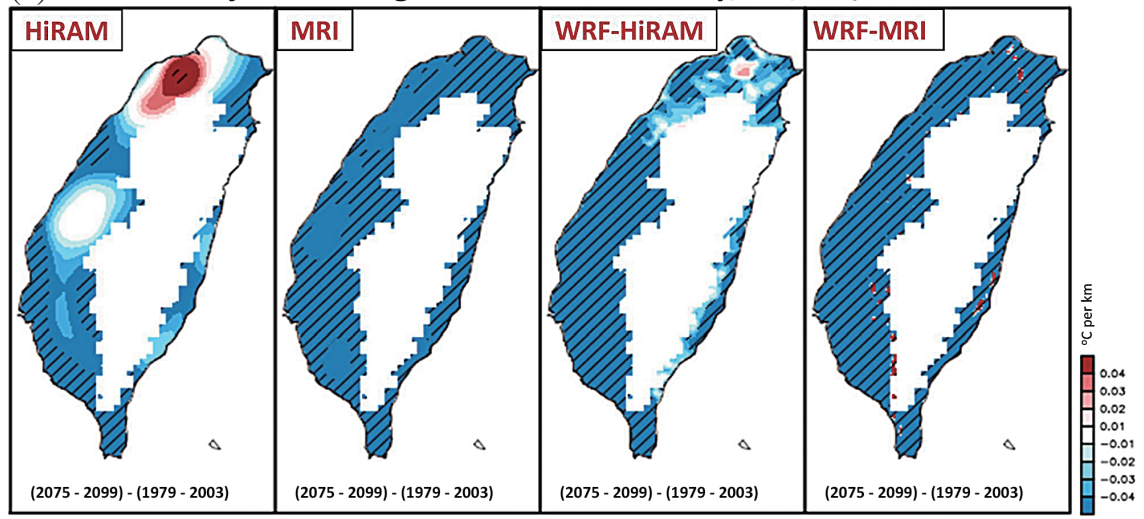

(b) Projected change in temperature area-averaged over Taiwan

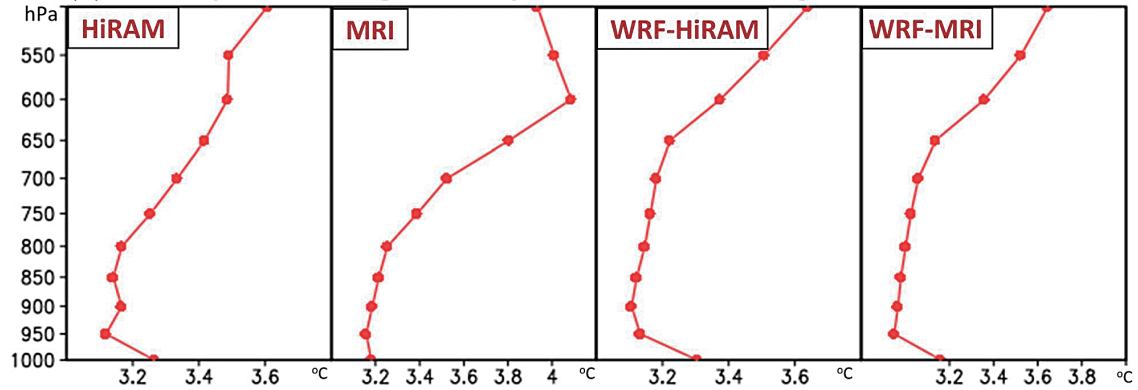

Fig. 12. Difference between future (2075 - 2099) and present-day (1979 - 2003) simulations for (a) the daytime thermal instability [i.e., -dT/dz (estimated from the temperature change with height, between 850 and $500 \mathrm{hPa}$ )] at $14 \mathrm{LT}$, and (b) vertical profile of temperature at $14 \mathrm{LT}$ area-averaged over Taiwan. The data were extracted from HiRAM, MRI, WRF-HiRAM, and WRF-MRI. In plot (a) the changes significant at the 90\% confidence interval are marked, and the mountain areas are blocked.

(also the distribution of CAR's contribution to total summer rainfall) over western Taiwan, but not over eastern Taiwan and the high-mountains (see Figs. 3 - 5).

The future projections from all models suggest that southwest Taiwan's CAR amount will decrease significantly in the future (Fig. 6). Such a decrease in southwest Taiwan's CAR amount is found to be induced mainly because of the decrease in CAR frequency (Fig. 8), not the change in CAR rate (Fig. 9). For the cause of future change in CAR frequency (see Figs. 10 - 11), analyses indicate that both the decrease in surface wind convergence (i.e., weaker dynamic lifting) and the decrease in thermal instability (i.e., weaker thermal lifting) lead to CAR frequency suppression over southwest Taiwan in the future. While for eastern Taiwan, where the projections show an increase in surface wind convergence (Figs. 10 - 11) but a decrease in thermal instability (Fig. 12), large differences were found among the models' CAR frequency projections (Fig. 8).

Note that even though the difference between HiRAM and MRI's simulations presented in this study are very large, the difference can actually be significantly reduced after dynamical downscaling using WRF driven by the global climate models. This implies that the WRF dynamical downscaling approach used in this study is not very sensitive to its driving forcing. However, it should be mentioned that the results of this study could be too dependent on the physical parameterization setup, as inferred from the review of regional climate model usage over complex topographic areas given by Lee and Hong (2014). Further studies on the sensitivity testing of physical parameterization, with increasing number of vertical levels employed in the models, are planned in the future to clarify this issue.

In Part II of this two-part investigation we will clarify if the method presented in this study can be applied to improve the CAR activity simulation over South China and Luzon (i.e., the two subtropical land areas closest to Taiwan). We will also examine the characteristics and causes of the projected changes in CAR activity over South China and Luzon. These examinations will help the local community better understand how regional climate will likely change in the future under global warming.

Acknowledgements The authors thank the Anonymous Reviewers for their comments and suggestions which greatly improved this manuscript. This research was supported by the Ministry of Science and Technology of Taiwan under MOST 103-2621-M-865-001, MOST 104-2621-M-865-001, MOST 104-2111-M-003-001, MOST 105-2119-M-003-002, and MOST 105-2625-M-003-002. H. H. Hsu was supported by MOST 100-2119-M-001-029 -MY5, and C. Y. Tu was 
supported by MOST 105-2111-M-001-001-MY2.

\section{REFERENCES}

Chen, C. S. and Y. L. Chen, 2003: The rainfall characteristics of Taiwan. Mon. Weather Rev., 131, 1323-1341, doi: 10.1175/1520-0493(2003)131<1323:TRCOT>2.0 .CO;2. [Link]

Chen, C. S., W. S. Chen, and Z. Deng, 1991: A Study of a Mountain-generated Precipitation System in Northern Taiwan during TAMEX IOP 8. Mon. Weather Rev., 119, 2574-2606, doi: 10.1175/1520-0493(1991)119<2 574:ASOAMG >2.0.CO;2. [Link]

Chow, K. C. and J. C. L. Chan, 2009: Diurnal variations of circulation and precipitation in the vicinity of the Tibetan Plateau in early summer. Climate Dyn., 32, 5573, doi: 10.1007/s00382-008-0374-x. [Link]

Collins, W. D., P. J. Rasch, B. A. Boville, J. J. Hack, J. R. McCaa, D. L. Williamson, J. T. Kiehl, B. Briegleb, C. Bitz, S. J. Lin, M. Zhang, and Y. Dai, 2004: Description of the NCAR Community Atmosphere Model (CAM 3.0). NCAR Technical Note NCAR/TN464+STR, National Center for Atmospheric Research, Boulder, Colorado, 214 pp.

Cressman, G. P., 1959: An operational objective analysis system. Mon. Weather Rev., 87, 367-374, doi: 10.1175/152 0-0493(1959)087<0367:AOOAS>2.0.CO;2. [Link]

Hong, S. Y. and M. Kanamitsu, 2014: Dynamical downscaling: Fundamental issues from an NWP point of view and recommendations. Asia-Pac. J. Atmos. Sci., 50, 83-104, doi: 10.1007/s13143-014-0029-2. [Link]

Hong, S. Y., J. Dudhia, and S. H. Chen, 2004: A revised approach to ice microphysical processes for the bulk parameterization of clouds and precipitation. Mon. Weather Rev., 132, 103-120, doi: 10.1175/1520-0493 (2004)132<0103:ARATIM>2.0.CO;2. [Link]

Hong, S. Y., Y. Noh, and J. Dudhia, 2006: A new vertical diffusion package with an explicit treatment of entrainment processes. Mon. Weather Rev., 134, 2318-2341, doi: 10.1175/MWR3199.1. [Link]

Huang, W. R. and J. C. L. Chan, 2012: Seasonal variation of diurnal and semidiurnal rainfall over Southeast China. Climate Dyn., 39, 1913-1927, doi: 10.1007/s00382011-1236-5. [Link]

Huang, W. R. and K. C. Chen, 2015: Trends in pre-summer frontal and diurnal rainfall activities during 1982-2012 over Taiwan and Southeast China: Characteristics and possible causes. Int. J. Climatol., 35, 2608-2619, doi: 10.1002/joc.4159. [Link]

Huang, W. R. and S. Y. Wang, 2014: Impact of land-sea breezes at different scales on the diurnal rainfall in Taiwan. Climate Dyn., 43, 1951-1963, doi: 10.1007/ s00382-013-2018-z. [Link]

Huang, W. R., J. C. L. Chan, and S. Y. Wang, 2010: A planetary-scale land-sea breeze circulation in East Asia and the western North Pacific. Q. J.R. Meteorol. Soc., 136, 1543-1553, doi: 10.1002/qj.663. [Link]

Huang, W. R., J. C. L. Chan, and A. Y. M. Au-Yeung, 2013: Regional climate simulations of summer diurnal rainfall variations over East Asia and Southeast China. Climate Dyn., 40, 1625-1642, doi: 10.1007/s00382-0121457-2. [Link]

Huang, W. R., H. H. Hsu, S. Y. Wang, and J. P. Chen, 2015: Impact of atmospheric changes on the low-frequency variations of convective afternoon rainfall activity over Taiwan. J. Geophys. Res., 120, 8743-8758, doi: 10.1002/2015JD023568. [Link]

Johnson, R. H. and J. F. Bresch, 1991: Diagnosed characteristics of precipitation systems over Taiwan during the May-June 1987 TAMEX. Mon. Weather Rev., 119, 2540-2557, doi: 10.1175/1520-0493(1991)119<2540: DCOPSO $>2.0 . C O ; 2$. [Link]

Kerns, B. W. J., Y. L. Chen, and M. Y. Chang, 2010: The diurnal cycle of winds, rain, and clouds over Taiwan during the mei-yu, summer, and autumn rainfall regimes. Mon. Weather Rev., 138, 497-516, doi: 10.1175/2009MWR3031.1. [Link]

Lee, J. W. and S. Y. Hong, 2014: Potential for added value to downscaled climate extremes over Korea by increased resolution of a regional climate model. Theor. Appl. Climatol., 117, 667-677, doi: 10.1007/s00704013-1034-6. [Link]

Lee, J. W., S. Y. Hong, E. C. Chang, M. S. Suh, and H. S. Kang, 2014: Assessment of future climate change over East Asia due to the RCP scenarios downscaled by GRIMs-RMP. Climate Dyn., 42, 733-747, doi: 10.1007/s00382-013-1841-6. [Link]

Liang, X. Z., K. E. Kunkel, G. A. Meehl, R. G. Jones, and J. X. L. Wang, 2008: Regional climate models downscaling analysis of general circulation models present climate biases propagation into future change projections. Geophys. Res. Lett., 35, L08709, doi: 10.1029/2007GL032849. [Link]

Lin, C. Y., Y. J. Chua, Y. F. Sheng, H. H. Hsu, C. T. Cheng, and Y. Y. Lin, 2015: Altitudinal and latitudinal dependence of future warming in Taiwan simulated by WRF nested with ECHAM5/MPIOM. Int. J. Climatol., 35, 1800-1809, doi: 10.1002/joc.4118. [Link]

Lin, P. F., P. L. Chang, B. J. D. Jou, J. W. Wilson, and R. D. Roberts, 2011: Warm season afternoon thunderstorm characteristics under weak synoptic-scale forcing over Taiwan Island. Weather Forecast., 26, 44-60, doi: 10.1175/2010WAF2222386.1. [Link]

Lin, P. F., P. L. Chang, B. J. D. Jou, J. W. Wilson, and R. D. Roberts, 2012: Objective prediction of warm season afternoon thunderstorms in northern Taiwan using a fuzzy logic approach. Weather Forecast., 27, 11781197, doi: 10.1175/WAF-D-11-00105.1. [Link] 
Mizuta, R., H. Yoshimura, H. Murakami, M. Matsueda, H. Endo, T. Ose, K. Kamiguchi, M. Hosaka, M. Sugi, S. Yukimoto, S. Kusunoki, and A. Kitoh, 2012: Climate simulations using MRI-AGCM3.2 with 20-km grid. J. Meteorol. Soc. Jpn., 90A, 233-258, doi: 10.2151/ jmsj.2012-A12. [Link]

Monin, A. S. and A. M. Obukhov, 1954: Basic laws of turbulent mixing in the surface layer of the atmosphere. Tr. Akad. Nauk SSSR Geophiz. Inst., 24, 163-187.

Ruppert, J. H., R. H. Johnson, and A. K. Rowe, 2013: Diurnal circulations and rainfall in Taiwan during SoWMEX/ TiMREX (2008). Mon. Weather Rev., 141, 3851-3872, doi: 10.1175/MWR-D-12-00301.1. [Link]

Skamarock, W. C., J. B. Klemp, J. Dudhia, D. O. Gill, D. M. Barker, M. G. Duda, X. Y. Huang, W. Wang, and J. G. Powers, 2008: A description of the Advanced Research WRF Version 3. NCAR Technical Note NCAR/ TN-475+STR, National Center for Atmospheric Research, Boulder, Colorado, USA, 113 pp, doi: 10.5065/ D68S4MVH. [Link]

Tewari, M., F. Chen, W. Wang, J. Dudhia, M. A. LeMone, K. Mitchell, M. Ek, G. Gayno, J. Wegiel, and R. H. Cuenca, 2004: Implementation and verification of the unified NOAH land-surface model in the WRF model. 20th Conference on Weather Analysis and Forecasting/16th Conference on Numerical Weather
Prediction, American Meteorological Society, Seattle, WA, US., 6 pp.

Von Storch, H. and F. W. Zwiers, 1999: Statistical Analysis in Climate Research, Cambridge University Press, 484 pp, doi: 10.1017/CBO9780511612336. [Link]

Wang, S. Y. and T. C. Chen, 2008: Measuring East Asian summer monsoon rainfall contributions by different weather systems over Taiwan. J. Appl. Meteorol. Climatol.,47,2068-2080,doi: 10.1175/2007JAMC1821.1. [Link]

Wang, S. Y., H. H. Chia, R. R. Gillies, and X. Jiang, 2013: Quasi-biweekly mode and its modulation on the diurnal rainfall in Taiwan forecasted by the CFS. Weather Forecast., 28, 981-993, doi: 10.1175/WAFD-12-00120.1. [Link]

Xue, Y., Z. Janjic, J. Dudhia, R. Vasic, and F. De Sales, 2014: A review on regional dynamical downscaling in intraseasonal to seasonal simulation/prediction and major factors that affect downscaling ability. Atmos. Res., 147-148, 68-85, doi: 10.1016/j.atmosres.2014.05.001. [Link]

Zhao, M., I. M. Held, S. J. Lin, and G. A. Vecchi, 2009: Simulations of global hurricane climatology, interannual variability, and response to global warming using a 50-km resolution GCM. J. Climate, 22, 6653-6678, doi: 10.1175/2009JCLI3049.1. [Link] 